\title{
NEUROSYPHILIS
}

\section{A Review of Recent Literature}

\author{
By W. D. NrCoL, M.B., F.R.C.P. \\ Physician and Lecturer in Psychological Medicine, Royal Free Hospital ; Physician Superintendent, Horton \\ Hospital, Epsom
}

In this article it is proposed to review some of the work which has been published during the last two years, more especially with reference to asymptomatic neurosyphilis, and the late clinical manifestations, general paralysis of the insane, tabes and optic atrophy.

\section{Asymptomatic Neurosyphilis}

By the term 'asymptomatic neurosyphilis' is meant the condition characterized by the presence of abnormalities in the cerebrospinal fluid due to syphilis, unaccompanied by any clinical signs or symptoms. It cannot be stressed too often that the earlier treatment is instituted the better the prospect of the arrest of the disease process, the best results of all being obtained in those cases brought under treatment in the asymptomatic stage.

Hahn and Clark (1946) have made a detailed analysis of 1800 patients treated at the Johns Hopkins Clinic between the years I9I4 and I94I. Out of these 1800 patients were selected 533 with at least two cerebrospinal fluid examinations and 467 with at least two physical examinations. Their work affords an excellent study, more especially with regard to the prognosis of neurosyphilis. The classification of the type of abnormality of spinal fluid is based upon that of Earle Moore. This grouping is of such paramount importance and so universally used in America, that it might be useful to recapitulate here what is meant. Group I fluid reveals a slight increase of cells 5-30, with normal protein content, a negative colloidal gold -curve and a negative Wassermann. In Group II the cells are similarly increased as in Group I, protein may be raised from 30 to 75 , the colloidal gold curve is variable and the Wassermann weakly positive, while in Group III fluid the cells are increased from Io to 200 , the protein from 50 to 200 , the Wassermann is strongly positive and the colloidal gold curve exhibits what is known as the paretic formula, the first five tubes being completely decolourized. These authors, in their analysis, make a sharp distinction between early and late asymptomatic neurosyphilis : the early form is confined to patients with a definite history of 2 years or less from the primary attack, while in the late type the lapse of time following the original infection must be more than 2 years. Of 533 patients only 8.5 per cent. had received any treatment prior to diagnosis of asymptomatic neurosyphilis, not one had received as many as a total of 40 injections of trivalent arsenic and heavy metal. The more strongly positive the spinal fluid on initial examination, the more unfavourable is the prognosis with regard to the final outcome. The longer the duration of the infection the more strongly positive and more resistant the cerebrospinal fluid. In respect of the relationship of the cerebrospinal fluid outcome to the clinical out come the authors state that the probability of de-e veloping clinical neurosyphilis at the end of five? years' observation was 16.2 per cent. in patients whose fluid became worse or remained unchanged, contrasted with only 3.1 per cent. in patients whose fluid became negative or significantly improved: by the tenth year these probabilities were 36.3 per cent. and 6.8 per cent. respectively. The probability of developing parenchymatous neurosyphilis increases directly in proportion to the severity of the initial spinal fluid involvement. It should be noted, however, that in this large series only 40 patients, over one-half of whom had an initial Group III fluid and most of whom proved resistant to other types of therapy, received malaria. Merritt (1945) presents a useful analysis of 200 cases of asymptomatic neurosyphilis : I IO men, 90 women. The sex incidence was relatively equal, which is in sharp contrast to that found in parenchymatous neurosyphilis, where the male is a much more frequent victim. Three quarters of the patients had Group I abnormalities in the spinal fluid ; none of these patients developed the parenchymatous type of disease and only 4 per cent. developed symptomatic neurosyphilis of the meningovascular type. In those patients who had Group III fluid, parenchymatous neurosyphilis developed in 20 per cent. In a much wider survey Merritt found the incidence of asymptomatic neurosyphilis present in 9.5 per cent. of 2263 
patients who gave a history of syphilis of five years or more. On the other hand, abnormalities in the cerebrospinal fluid are increasingly prevalent, reaching a peak incidence of 35 per cent. of all syphilitics 18 months after the original infection. Treatment recommended is firstly so-called routine with trivalent arsenic and bismuth, secondly tryparsamide, and thirdly fever. In patients who have received 30 or more injections of arsenic, and in whom the findings of the spinal fluid remain abnormal, recourse to other methods should be immediately sought.

\section{General Paralysis of the Insane}

Arieti (I945) gives a critical review of the literature of senile G.P.I. together with a clinical and pathological description of six cases. Patients in whom symptoms appear for the first time at the age of 60 or over are regarded as being senile cases of general paralysis. The incidence for the decade 1932-1941 in the New York State hospital was 10.58 per cent.; there was a tendency for the numbers to increase and the disproportion between male and female was even more pronounced in senility. The symptomatology was that of an organic psychosis and neurological signs were indistinguishable from those seen in cerebral arteriosclerosis. The irregularity of the findings of the cerebrospinal fluid makes diagnosis difficult; the course of the disease is rapid, with progressive deterioration.

Reynolds and Moore (1946) in a review of recent literature on syphilis refer to a point of diagnostic interest in general paralysis. Thompson describes the palmomental reflex, which is elicited by stroking the thenar eminence from the base of the thumb to the wrist: in a positive response the skin on the chin on the ipsilateral side near the mid line dimples and moves laterally and upwards. This phenomenon, he claims, is one of the first physical signs to appear and though occurring sometimes in other neurological disorders, it is far more common in the paretic.

\section{Electroencephalogram in Neurosyphilis}

Greenblatt and Levin (1945) describe the electroencephalographic abnormalities met with in neurosyphilis. Investigations were carried out in 330 cases, of whom 233 were selected; against this group was a control of 240 from medical, nursing and other auxiliary hospital staff. Amongst the latter group were recorded io per cent. abnormal E.E.G.s. In the neurosyphilitic series 50 per cent. E.E.G.s were abnormal ( 46 per cent. abnormal slow ; 44 per cent. abnormal fast, and Io per cent. paroxysmal). The incidence of abnormality according to clinical types was highest in meningovascular syphilis (60 per cent.), in general paresis it was 55 per cent., optic atrophy⿳亠㐅⿸厂 44 per cent., and tabes I 4 per cent. ; the tabetics were not significantly different from the control ${ }^{\circ}$ group. It is noted, however, that 20 per cent. of the total gave positive histories of cerebral seizures. $\overrightarrow{\vec{S}}$ In the group of general paretics the highest incidence of abnormal E.E.G. occurred in those cases with seizures, the next highest in the group $\overline{\bar{s}}$. with marked tremors and dysarthria, so that thereচ is some correlation between the E.E.G. abnormality and the clinical condition. No correlation wass found with regard to the presence of pupillary $\vec{O}$ changes, delusions or hallucinations. The high $\overrightarrow{-}$ incidence of abnormality found in optic atrophy is a mystery. These authors found that the healingo of these brain lesions after therapy reflected an3 improvement in the electrical pattern of the E.E.G.i Callaway and his co-workers (1945) report the study of E.E.G. before and after penicillin therapy in 38 patients ( 33 general paresis and taboparesis). in They agree that improvement in the E.E.G. is useful in determining the influence of penicillin ${ }^{3}$ therapy, but there is no correlation between the $\vec{c}$ severity of the neurosyphilitic disorder and the presence or absence of E.E.G. abnormalities. They, advanced the theory that the abnormality is an ' expression of local anoxia and a generalized $\operatorname{gr} \vec{\bullet}$ localized cerebral inflammation.'

\section{Malaria Therapy}

A debt of gratitude should be accorded to Bruetsch (1946) for releasing a document written by Wagner-Jauregg in 1935 on the 'History of the Malaria Treatment of General Paralysis,' which $\stackrel{\circ}{\overrightarrow{7}}$ hitherto had not been published. This is a docu- $\overrightarrow{\vec{B}}$ ment of considerable historical interest. WagnerJauregg describes how he had the idea of giving mental patients malaria or erysipelas as far back: as 1887. The idea was not pursued as far as $\frac{}{3}$ malaria was concerned; erysipelas was tried with:poor effect, but in any case was not given to any 3 paretics. He then turned his attention to Tuberculin and reported in 1909 with great caution some moderate success in cases of general paralysis ;o moreover he maintained that the elevated temperature was not the fundamental factor in treatment. Tuberculin treated cases were liable to relapse and again Wagner-Jauregg was attracted by the ideao of using malaria. It was in June 1917 that "an sign of destiny' presented itself. A woundedN soldier returned from the Balkan front had an $\omega$ attack of malaria and this was reported by WagnerJauregg's assistant, quinine was withheld, the diagnosis confirmed, and the blood of this soldieres was used for scarifying the arms of three general ${ }_{D}^{+}$ paralytics ; two developed fever, and so the firsto cases on record received malaria therapy.

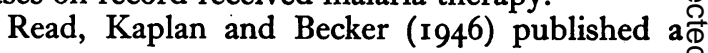


useful paper dealing with complications during malaria therapy. The species of malaria employed were Benign Tertian (P. Vivax) and Quartan (P. Malariae). Their analysis comprises 300 cases, of whom 2 I I received Benign Tertian, the remainder being inoculated with quartan malaria. The chief complications referred to are jaundice, oedema, and mild renal damage. Jaundice in 50 per cent. of cases appears to be due to excessive haemolysis. This is of interest and is a pointer to the need for carrying out parasite counts on patients during malaria therapy. The haemolysis is undoubtedly the result of a heavy parasitaemia. At the Horton Malaria Therapy Centre, where a series of over 2000 cases have been treated, it has occurred very rarely. This is probably attributable to the fact that when parasite counts, which are done daily, show a high parasitaemia, fever is temporarily interrupted. Care is needed in arresting other manifestations such as oedema and renal damage. Other complications referred to are very rare, such as purpura, urticaria, hyperlipaemia and spontaneous splenic rupture. Reference is also made to two cases exhibiting an unusual form of severe respiratory distress with cyanosis, both being relieved by transforming a quotidian type of fever into a true tertian by the administration of thiobismol. No mention is made of those alarming cases, fortunately few, who show signs of peripheral failure. The use of Thio-Bismol in malaria therapy was first described in America by Schwartz (1939) and in this country by Whelen and Shute (1943). The action of this drug in converting quotidian fever into tertian in a very high proportion of cases treated with benign tertian malaria is of great therapeutic importance, and in our experience has rendered the treatment comparatively safe. Kaplan and others (1946) have reported its use in Quartan malaria. There is much work to be done in this direction, but they make the interesting observation that the usual maximum dose of $0.2 \mathrm{gm}$. was ineffective in cases showing an unusually high parasitaemia.

From the Malaria Therapy Centre at Horton Nicol (1946) made a careful comparison between the results of malaria plus tryparsamide and malaria alone. Each group comprised 217 cases, 127 men and 90 women. With the exception of 44 taboparetics, all patients were suffering from general paralysis. The follow up extended over ten years and results were assessed clinically and serologically. Definitely better results were obtained in cases treated by malaria plus tryparsamide than by malaria alone. The trial of penicillin was advocated as being possibly superior to and less dangerous than tryparsamide.

\section{The Serological Follow Up}

From the same Centre, Whelen ( 1946$)$ reported a detailed analysis of the serological follow up of over 500 cases. The work was based largely on Dattner's premises, so well advocated in his book 'The Management of Neurosyphilis' (1944). It was found that most cerebrospinal fluids following malaria therapy became negative within three years from the end of treatment. The condition of the fluid at the end of the first year was a fairly reliable indicator as to whether further treatment was necessary or not. In over 90 per cent. of patients showing two consecutive 'negatives' at an interval of six months or more, the spinal fluid remained persistently negative-a point of immense practical importance. The necessity for educating the patient to the need for serial lumbar punctures following treatment is so universally recognized now, that every effort to minimize any after effects is essential.

L. J. Underwood (1946) presented a statistical analysis of 500 punctures. The incidence of post-lumbar puncture headache varied between I per cent. and 40 per cent. of punctures according to different workers. This wide range is difficult to explain, as also is the report that cisternal puncture (Kulchar) reduces the frequency of headaches. Solomon has used Extract Pituitary I c.c. intramuscularly and relieved many patients. Guttmann is reported to have produced complete relief with Ergotamine tartrate in 82.9 per cent. of 35 patients. In the report of Underwood, where a 22 gauge spinal needle was used, and 2 c.c. of 2 per cent. novocaine locally, the average amount of fluid collected being 6-8 c.c. incapacitating headaches occurred in 19 per cent. of cases. He makes the important observation that rest in bed after the spinal puncture did not reduce the incidence of post-lumbar puncture headache. At the Horton Clinic, where the Dattner needle with Harrison's modification (Down Bros.) has been employed for over Io years, post-lumbar puncture headache has only been encountered very rarely. We attribute this largely to the practice of allowing patients to leave the outpatient clinic almost immediately and not encouraging them to lie down afterwards. Col. A. J. King, in a personal communication, referred to this important point. He experienced considerable difficulty in Army clinics in Italy during the recent war, but found the incidence of post-lumbar puncture headaches fell dramatically directly the recumbent position after the spinal puncture was discontinued. This bears out Levin's experience (U.S. Army Medical Department) that by keeping patients active only 15 patients ( 0.67 per cent.) reported severe headaches in a series of 2237 lumbar punctures; he put forward the theory that intracranial hypotension 
and lowered spinal fluid pressure and hypertension with resultant headache are overcome by moderate exercise.

\section{Penicillin}

For the past two years the literature on neurosyphilis, as indeed in many other branches of medicine, has been dominated by penicillin therapy.

\section{The Action of Penicillin :}

Frazier and Frieden (1946) review the available evidence and the theories put forward as to the mode of action of penicillin on the spirochaete. Both experimental and clinical observations are discussed. Experiments in vivo are reported by Dunham and Rake, who put forward the theory that penicillin probably destroys the reproductive capacity of the spirochaete without destroying its power 'of motility. Frazier and Frieden themselves found changes in the structure of spirochaetes as a result of penicillin therapy. The length of the spirochaete altered considerably, there being an increase in long forms ( 14 to 22 microns); these long froms were less motile than the short forms, an indication in their opinion of excessive growth of the organism eventually leading to its death. Though these initial studies were confined to reports on only 5 patients under treatment for syphilis, the findings were constant.

Another vexed question has been the problem of the transfer of penicillin into the cerebrospinal fluid. McDermott and Nelson (I945) ask whether it is necessary in the treatment of neurosyphilis for an effective concentration of any particular chemotherapeutic agent to be present in the cerebrospinal fluid. They argue that there is no proof that an effective concentration of penicillin is not achieved in the tissues of the central nervous system merely because little or no penicillin is detectable in the cerebrospinal fluid, following parenteral administration. Using dilution techniques of bioassay these workers found no penicillin demonstrable in the spinal fluid from 70 patients being treated for neurosyphilis. On the assumption that in neurosyphilis, even where meningitis is present, the rate of infection is relatively slow, the continued presence of minute amounts is probably all that is necessary for effective therapy. This is in contrast to the more acute rapid purulent form of meningitis of nonspecific origin, where parenteral administration only might prove dangerously inadequate.

\section{Dosage of Penicillin}

In the early reports of the American clinicians the dosage in neurosyphilis was in the region of 2 mega units, but the general consensus of opinion now is to give at least 4 mega units. Reynolds anc Moore (1946) in their review of recent literature quote Raiziss' experiments in rabbit syphilis, where syphilitic infections were cured by 40,00 Oxford units of penicillin per kilogram bodkg weight over a period of 8 days. On this basis fo a human adult of $60 \mathrm{~kg}$. weight the total dose re흘 quired would be 2.4 mega units. Since, how ever, larger doses produced more rapid healing these workers recommended doubling the dose making a total of 4.8 mega units. Rose and Solomon (1947) draw the conclusion that 3 mega units of penicillin, supplemented by a short course of therapeutic fever, are insufficient in the treate ment of late neurosyphilis. The reason for the assertion was the need for re-treatment in one. third of their cases after one year's follow up an since 1945 they have recommended a course of mega units of penicillin.

\section{Methods of Administration}

The usual method is by intramuscular injection, 3 hourly throughout the 24 hours, in 50,000 unit doses for a period of 7 to ro days. Weickhard (1946) describes 5 cases where introthecal pen cillin was given, doses being gradually increase from 25,000 to 100,000 units; he maintains thas though in the experimental stages the methos is safe. This author quotes reports from ot workers, which are certainly not encouraging. F view of the excellent results obtained by the in tramuscular route, it would appear that intrathecos administration is unnecessary, if not harmfu品 Even intramuscular injections at repeated intervals throughout the 24 hours must be very trying fo the patient. In this country Lloyd Jones (1946) and his colleagues obtained good results in tho treatment of primary and secondary syphilis wit intramuscular injections (300,000 units) once. daily. Similarly Lourie (1945) and others advocate ambulatory treatment of early syphilis by three doses of 600,000 units at hourly intervals give 8 on 5 successive days. In the treatment of the later manifestations of neurosyphilis it has beeg the practice at the Horton Malaria Therapy Centre to give intramuscular penicillin, 300,00 units, once a day for 14 days, making a total of 4.2 mega units. In the work referred to above, penicillin was administered in aqueous solution Kirby and others (1945) discuss the use of pento cillin in beeswax peanut oil, with a view to delaying its absorption. They used a mixture of 4.8 pees cent. beeswax and 300,000 units of penicillin per cubic centimetre daily for 8 days. The authos considered the method safe and there was evidenced that the action of the drug was prolonged. Febrile reactions accompanied by convulsions have been reported by various workers in association wit 
penicillin therapy. These phenomena are generally accepted as Herxheimer reactions. Callaway (1946) tried to minimize the effects by the use of graduated doses of penicillin; patients received 5 injections of 10,000 units, after which they were given 79 consecutive injections of 50,000 units. In spite of this febrile Herxheimer reactions were frequent, but were not sufficiently severe to interfere with the completion of the course of therapy. Koteen and his colleagues (1947), using 4 mega units (25,000 units intramuscularly 2 hourly through the 24 hours), report febrile reactions in I 7 patients out of a total of I I I under review. Two tabetics died within two weeks of treatment. In a communication to a joint meeting of the French and British neurologists, held in April 1947, at the Royal Society of Medicine, Nicol and Whelen (1947 in the Press) reported their limited experience of the use of penicillin in the Horton Malaria Therapy Centre. In a series of 60 cases of general paresis so treated, no Herxheimer reactions were encountered, nor was any preparatory course of a heavy metal and potassium iodide given.

\section{Results of Penicillin Therapy}

It is indeed fortunate that several clinics have confined themselves to the use of penicillin alone, so that we are at any rate in some position to evaluate the efficacy of this drug, without having results masked by the supplementary use of malaria or other fever therapy. Results may be grouped under two headings-clinical and serological.

Clinically there seems to be general agreement that patients gain weight and feel quite different in themselves. Heyman (1947) refers to the subjective improvement, especially the asymptomatic group, who described themselves as ' one hundred per cent. different.' Nicol and Whelen (vide supra) reported the marked clinical improvement, particularly in patients who were confused and in poor physical condition, seen during the actual course of penicillin, . a phenomenon rarely encountered during malaria therapy. The improvement in the tabetic is difficult of assessment due to the large subjective element. Gammon and Stokes (vide supra) report on lightning pains in 40 tabetic and taboparetic patients. Some degree of improvement following penicillin therapy was noted in 82 per cent. ; on the other hand, 4 of $I_{5}$ patients in whom no pain had been present, developed pain during and after penicillin, which the authors attribute to a Herxheimer reaction. Koteen (1947 : vide supra) and his colleagues report considerable improvement of lightning pains in 17 out of 24 patients and also improvement of ataxia in half the series; no improvement of gastric crises was observed. These same workers record a striking improvement in a case of severe optic neuritis, who before receiving penicillin, had had intensive chemotherapy without interruption for the previous five years.

Serological results are of much easier assay and afford more accurate data as to the efficacy of penicillin therapy. O'Leary, Brunsting and Ockuly (1946) are unconvincing regarding the use of penicillin and exercise extreme caution in advocating the use of penicillin alone. This certainly does not seem to be the view held by other workers. Gammon and Stokes ('́946) used penicillin alone in 16r cases of neurosyphilis ; since May 1945 the dose employed was 4.8 mega units. Decision to retreat was based on failure of spinal fluid to become normal, failure of reversible symptoms to respond and a serological relapse. They advocate penicillin as the first choice of treatment for neurosyphilis, though this statement may be qualified in the case of severe paretics. Koteen (1947) and his co-workers are satisfied with 300,000 daily (in 25,000 unit doses) over a two week regimen and conclude that an increase of the daily total dosage would not increase the effectiveness of the therapy. They do, however, raise the question as to whether penicillin plus fever therapy might not be superior to the use of either agent alone. Nicol (1946) recommended the substitution of penicillin for tryparsamide as an adjuvant to malaria therapy in the treatment of parenchymatous forms of neurosyphilis. Rose and Solomon (r947) in a useful summary of a follow up of roo cases over one year or more give a comparison of different modes of therapy ; penicillin plus malaria, penicillin plus fever cabinet, and penicillin alone. Where penicillin was supplemented by other therapy, the amount of malaria was reduced to 4 to 6 paroxysms of fever and the time in the fever cabinet to 20 hours at $105^{\circ} \mathrm{F}$. The amount of penicillin given was 3 mega units at first, but since 1945 penicillin dosage was increased to 6 mega units. In most cases the cell count returned to normal earlier than the protein content, a decrease in the Wassermann occurred but was less rapid. Results generally were most favourable with penicillin plus malaria ; to quote these authors, they write ' after two years experience with penicillin our enthusiasm has been tempered and we have become aware of the limitations of this treatment as well as the advantages.'

Heyman (1947) in a follow up of 141 cases treated with doses of penicillin ranging from $\mathrm{I} .2$ to 6 mega units reports that with the use of 4 mega units penicillin will arrest the neurosyphilitic process in 85 per cent. of cases. He adds a cautious note, however, that longer periods of 
observation are necessary before evidence of serological relapse becomes evident. The criterion of relapse was based entirely on the cell count. For early and late asymptomatic neurosyphilis penicillin therapy is probably adequate, provided a careful follow up is observed. In late symptomatic neurosyphilis penicillin is not recommended as replacing fever therapy.

The introduction of penicillin to the armentarium against syphilis and its later manifestations has produced a spate of literature and it is impossible in our present state of knowledge to be dogmatic as to its use. Earle Moore and Mohr (1946) in a review of 9 patients with early and late asymptomatic syphilis, while their results were encouraging (only 4 patients had a serological relapse within the limits of a six month to one year's follow up) sound a note of warning and state that a minimum of five years' study and the treatment of many more patients are necessary before we can reach any definite conclusion. Dattner (1947) is of the opinion that so far penicillin, when given in adequate doses, is at least as effective in the treatment of neurosyphilis as combined fever and specific therapies. He adds a word of caution, however, in that we are ignorant of the "optimal relationship of dose and time to obtain the maximal therapeutic success.' While penicillin may well supersede other forms of therapy in the treatment of asymptomatic neurosyphilis, is it going to displace malaria or artificial fever therapy for late symptomatic neurosyphilis, more especially general paresis? At present, opinions of various observers are conflicting. Stokes, Steiger, and their co-workers (I946) look forward to the replacement of malaria therapy by penicillin; their observations are based on the follow up of 283 cases of neurosyphilis. Reynolds, Mohr and Earle Moore (1946), on the other hand, advocate the use of concurrent penicillin-malaria as the treatment of choice for cases of general paresis. Rose, Trevett and others (1945) in a series of 106 patients with symptomatic neurosyphilis, employing penicillin plus malaria or fever cabinet, reduced by half the amount of fever usually accepted as optimal.

It was several years before malaria was accepted as an established therapeutic measure ; it is now 3o years since Wagner-Jauregg introduced it, and it is still in vogue as the treatment par excellence for certain types of neurosyphilis. Penicillin is full of promise, but it will be many years before a definite conclusion can be arrived at. We have yet to find the optimum dosage, whether it should be given concurrently with malaria, or before $\frac{0}{0}$ supplementary to it, or, indeed, without malari at all.

\section{Conclusions}

If our therapeutic endeavours could be confined to the asymptomatic cases of neurosyphilis, the later crippling disabilities of symptomatic neurgr syphilis would never be witnessed. The soci and economic implications of general paresis alone are almost without bounds. Iskrant (1945) gives an estimate of the cost to the community of hospitalization of paretic patients in institutions it New York State and the consequent loss or ing pairment of productive capacity. When one learres that there is an estimated annual loss of workin years of life due to general paresis amounting 75,729 man years and approximately 112 million dollars loss in income, it is indeed time for og vigorous campaign to enable every possible means of antisyphilitic prophylaxis to be carried ouf? While these patients present themselves for treat. ment, it is incumbent on us to make every economic use possible of our discharges as advocated. by Whelan and Bree (1946) who make a plea for a wider conception of the promotion of optimum health.

BIBLIOGRAPHY
ARIETI, S. (1945), Am. Fourn. Psych., I01, 585.

BRUETSCH, WALTER J. (1946), F. $A . M . A .$, r3o, 14.

BRUETSCH, WALTER J. (1946), Am. F. Psych, बें CALLAWAY, J. L., et al. (1946), Am. F. Syph., 30, 110.

DATTNER, B. (1947), Arch. Neur. \& Psych., 57, 270.

FRAZIER C. N and FRIEDEN, E. H. (1946), $\mathcal{F} A M$. $A$. GAMMON, G. D., STOKES, J. H., et al. (1946), Ann. Int. Med,

GREENSBLATT, M. and LEVIN, S. (1945), Am. F. Psych

HAHN, R. D. and CLARK, E. G. (1946), Am. F. Syph., 30, 513. HEYMAN, A. (1947), Am. F. Med. Sci., 2r3, 66r.

ISKRANT, A. P. (1945), 手. Ven. Dis. Inform., 26, 175.

KIRBY W. M. M et al (I945), $A$. $A$., 26, 175

KOTEEN, H. DÖTY, E. J., et al. (1947), Am. $\mathscr{f}$. Syph., 31, 1.

LLOYD JONES, T. R., et al. (1946), Brit. Med. $\mathscr{F} .$, r, 567.

LOURIE, E. M., et al. (1945), Lancet ii, 696 .

LOWENBACH, H., et al. (I945), ₹.A.M.A., 129, 938. 29, 403.

MOORE, J. E. and MOHR, C. F. (1946), Am. F. Syph., 30, 405.马

NICOL, W. D. (1946), Brit. Fourn. Ven. Dis., 22, I 12. . 30, 405.3. (in press).

O'LEARY, P. A., BRUNSTIING, L, A. and OKULY, O. (1946) F.A.M.A., 130, 608 .

READ, H. S., KAPLAN, E. and BECKER, F. T. (1946), Anin. Int. Med.' $24,444$.
EYNOLD, F. W., MOHR, C. F. and MOORE, J. E. (194 REYNOLDS, F. W., MOHR, C. F. and MOORE, J. E. (I94)
F.A.M.A., I31, 1255 .
REYNOLDS, F. W. and MOORE, J. E. (1946), Arch. Int. Meq
78,600 and 739 . ROSE, 609 and 739 . and SOLOMON, H. C. (1947), F.A.M.A., 133, 5. O ROSE, A. S. and TREVETT, L. D. (1945), Am. F. Syph., 29, 48爪⿱

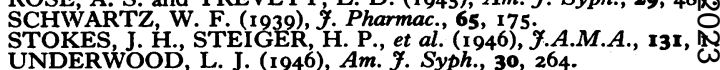
UNDERWOOD, L. J. (1946), Am. F. Syph., 30, 264.

WEICKHARDT, G. D. (1946), $A m$. $\mathscr{F} . S y p h ., 30,23$.

WHELEN, M. (1946), Brit. Fourn. Ven. Dis., 22, 121.

WHELEN, M. and BREE, M. H. (1946), Lancet ii, 477. WHELEN, M. and SHUTE, P. G. (1943), F. Trop. Med. aff
Hyg., 46, I, I.

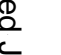

\section{.}

